\title{
UN MANUSCRIT DE «L'INTERNATIONALE»
}

C'est quelques mois avant sa mort qu'Eugène Pottier remit à son éditeur le texte de L'Internationale destiné à être publié parmi les Chants révolutionnaires (Paris 1887). Il le date alors: Paris, juin 1871. Ce poème étant devenu, avec l'appoint de la musique de Pierre Degeyter, le plus célèbre de tous ceux de Pottier, il semble étonnant que celui-ci ait attendu seize ans pour le rendre public alors qu'il en a publié tant d'autres pendant son exil et surtout après l'amnistie. Il est certain que cet étonnement est surtout fonction de l'importance prise par L'Internationale bien après sa publication et la disparition de son auteur; le fait est que Pottier n'a pas inclus L'Internationale parmi ses Poésies d'économie sociale, éditées en 1884 , et qu'il ne l'a pas plus proposée à son ami Argyriadès à qui il a remis plusieurs poèmes inédits pour publication dans La Question Sociale.

Or voici que le manuscrit de L'Internationale que détient l'Institut international d'Histoire sociale peut fournir une explication: ce texte, qui est assurément une version antérieure à celle éditée en 1887, n'avait peut-être pas été jugé satisfaisant par le poète; il l'a considérablement modifié avant de se décider à le faire publier. Ce manuscrit de quatre pages, signé: E. Pottier, figure dans la collection Descaves (dossier Pottier); Lucien Descaves l'avait acheté, avec cinq autres, à Henrik Barsen qui les tenait de sa femme, petite-nièce de Pottier.

Sa reproduction fac similé et sa comparaison avec le texte de 1887 permet de faire quelques constatations. Le manuscrit ne comporte aucune rature et n'est pas daté; rien ne permet d'affirmer que nous sommes en présence du texte original de juin 1871 - si toutefois en ce mois qui suit la Semaine sanglante Pottier, alors caché dans Paris, a bien écrit une Internationale en six strophes, comme le veut la tradition... Il semble qu'il y ait eu une élaboration plus lente qui, en passant par le manuscrit d'Amsterdam, aboutit au poème publié en 1887.

Si le refrain est identique dans les deux versions, les couplets présentent des variantes, parfois importantes, ou sont même totalement différents. Le texte de"1887 est suffisamment répandu?pour.que nous 
le supposions connu: chacun peut donc juger des transformations apportées par l'auteur.

L'idée du premier couplet - le plus souvent chanté - est identique, mais il n'y avait pas encore les vers célèbres (bien que n'étant pas des meilleurs):

«La raison tonne en son cratère,

C'est l'irruption [éruption] de la fin.

Du passé, faisons table rase, $[\ldots]$

Le monde va changer de base."

Le deuxième couplet, commun aux deux versions, exprime à peu près dans la même forme la thèse de la Première Internationale: "L'émancipation de la classe ouvrière doit être l'œuvre des travailleurs euxmêmes." Le troisième couplet est devenu le cinquième dans la version définitive; si les quatre premiers vers - identiques - peuvent avoir été écrits au moment de la Commune, les quatre derniers de la version 1887 de ce "couplet des généraux" antimilitariste se conçoivent difficilement dans le contexte de la répression versaillaise:

«S'ils s'obstinent, ces cannibales,

A faire de nous des héros,

Ils sauront bientôt que nos balles

Sont pour nos propres généraux!» ${ }^{1}$

En revanche, le quatrième couplet du manuscrit - rejeté ensuite par Pottier - baigne dans l'atmosphère de la Semaine sanglante. Pottier n'a apporté que quelques retouches de style au cinquième couplet dont il fait le dernier en 1887; il apporte ainsi à son poème une conclusion politique bien supérieure aux vœux pieux de l'ancien sixième couplet qu'il a justement éliminé. Remarquons aussi que les troisième et quatrième couplets de la version imprimée (respectivement: «L'Etat comprime et la loi triche...» et "Hideux dans leur apothéose...») sont entièrement nouveaux par rapport au manuscrit d'Amsterdam.

Tout ceci nous amène à conclure que si ce manuscrit n'est peut-être pas le texte primitif que Pottier aurait écrit en juin 1871, il est beaucoup plus proche de la Commune que celui publié en 1887. Eugène Pottier ne le considérait sans doute pas suffisamment au point pour être publié sous cette forme pendant son exil et, ensuite, devenu militant du Parti ouvrier, il jugea nécessaire de le remanier, pour en faire le chant de lutte et d'espoir que l'on connaît.

1 On pourrait y voir cependant un rappel - conscient ou inconscient - de la mort des généraux Lecomte et Thomas. 
586

DE LA LITTERATURE A L'HISTOIRE

Pimtecuaterinales.

c'est to Plute furate.

S giompan mon el demasio
ginternationale.

Sera be geare hi..... in..

Wolrout! f'anne) du jootltaire' ' Giavaullecass, gioupinen neres exfir.

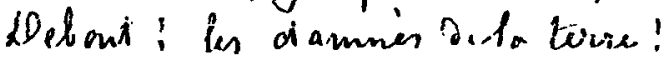

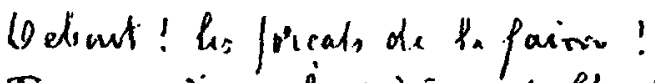

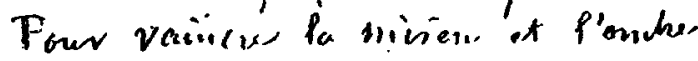
"Goule. estave, Jelent! Debent!

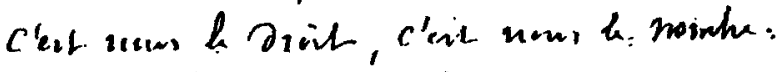

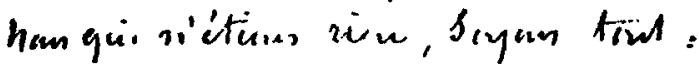

S'as lalutu fircule,

gimpian-sumn of Jemair.

Pigiternationales

derev le geene hurnaion:

if "ist pord. Sawrenes supriemes:

hi dien, hi cesar, ni tichliune.

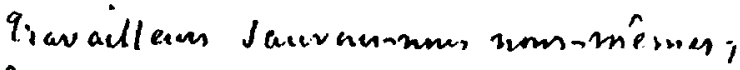

Giarachline ain Jalut comonum.

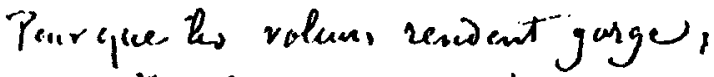

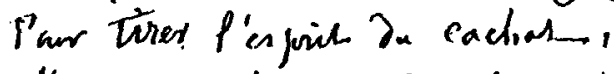

allinniares nothe graside. forgu!

Battan be fer quaind a est chand:

https://doi.org/10.1017/S0020859000006726 Published online by Cambridge University Press 
"L'INTERNATIONALE》

587

cim 1. latte jomal.

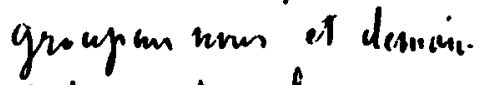

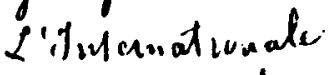

Serale yeme hurira.er

Ouvien, paysan, , 11ress Joinnies fa grand parti ice traveillems,

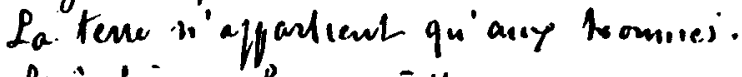
Liorif ira Pager aillears.

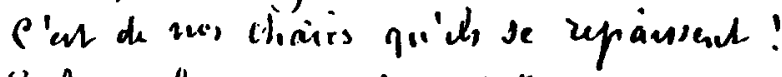

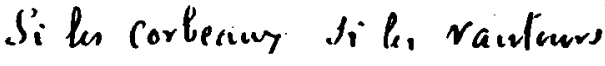

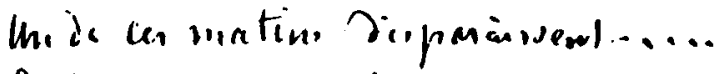

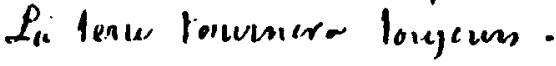

C'ell lo luth imale gioupan now et Dnuair

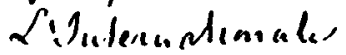
Jerale geve humain

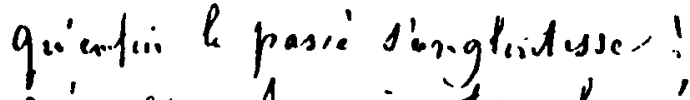

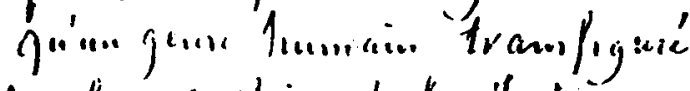
Smile cod elair de to. Jestices micicise aree t'epi zo'ke!

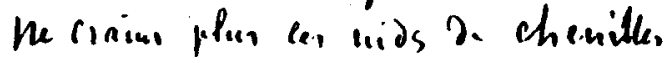

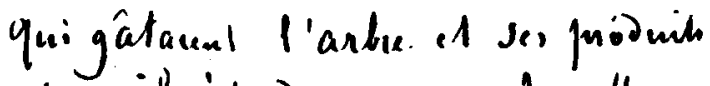
lhavail ietends sur noi jabillle.

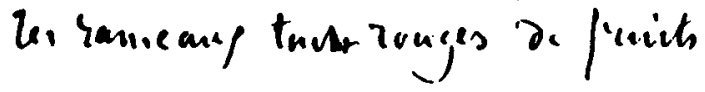

https://doi.org/10.1017/S0020859000006726 Published online by Cambridge University Press 
588

DE LA LITTERATURE A L'HISTOIRE

c'ear b lutte furah

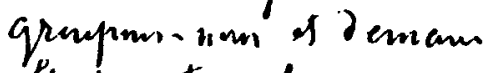

Pimilesiationale,

seva le geure hurian..

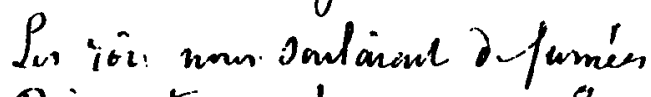

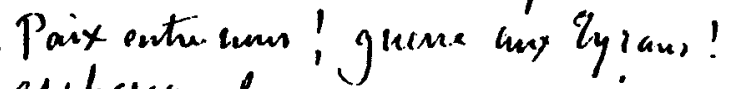
appliglociu la greve Aut arinces Ciotze un l'aur! st ionpm, ke, iangs! Baride, frimes explaction on pricher qui vit de f.homomes enc crimnosed;

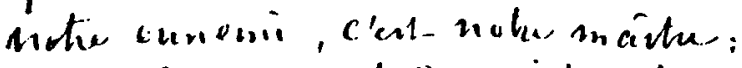
volí l. liat riaje etermel.

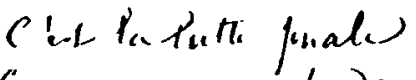

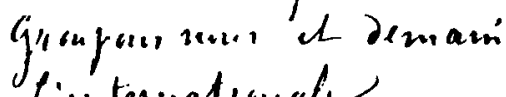

fieterinationale,

Serak geme tursicine.

P'paguenge encer va nous fordus:

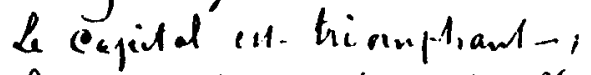

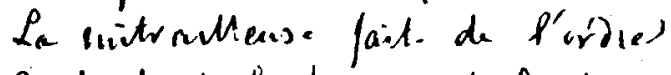

9.r. hachant to tennies at P'enfaul.

l'Tlsure,folle dac der coline.

Sur taus Caiduries calciué,

Soude. a fo grever des Salaine!

degreve dar assossilies.

https://doi.org/10.1017/S0020859000006726 Published online by Cambridge University Press 
"L'INTERNATIONALE»

589

C'at L-lube /male

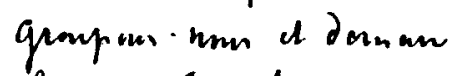
Licutionationale.

Senale geme hemian

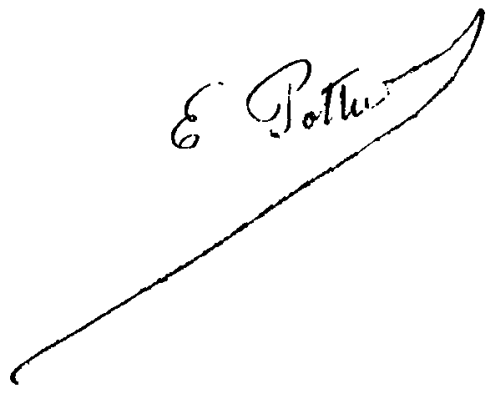

https://doi.org/10.1017/S0020859000006726 Published online by Cambridge University Press 